\title{
Actual and undiagnosed HIV prevalence in a community sample of men who have sex with men in Auckland, New Zealand
}

\author{
Peter JW Saxton ${ }^{1 *}$, Nigel P Dickson', Richard Griffiths ${ }^{1}$, Anthony J Hughes ${ }^{2}$ and John Rowden ${ }^{2}$
}

\begin{abstract}
Background: The prevalence of HIV infection and how this varies between subgroups is a fundamental indicator of epidemic control. While there has been a rise in the number of HIV diagnoses among men who have sex with men (MSM) in New Zealand over the last decade, the actual prevalence of HIV and the proportion undiagnosed is not known. We measured these outcomes in a community sample of MSM in Auckland, New Zealand.

Methods: The study was embedded in an established behavioural surveillance programme. MSM attending a gay community fair day, gay bars and sex-on-site venues during 1 week in February 2011 who agreed to complete a questionnaire were invited to provide an anonymous oral fluid specimen for analysis of HIV antibodies. From the 1304 eligible respondents (acceptance rate 48.5\%), 1049 provided a matched specimen (provision rate 80.4\%).

Results: HIV prevalence was 6.5\% (95\% Cl: 5.1-8.1). After adjusting for age, ethnicity and recruitment site, HIV positivity was significantly elevated among respondents who were aged 30-44 or 45 and over, were resident outside New Zealand, had 6-20 or more than 20 recent sexual partners, had engaged in unprotected anal intercourse with a casual partner, had had sex with a man met online, or had injected drugs in the 6 months prior to survey. One fifth (20.9\%) of HIV infected men were undiagnosed; $1.3 \%$ of the total sample. Although HIV prevalence did not differ by ethnicity, HIV infected non-European respondents were more likely to be undiagnosed. Most of the small number of undiagnosed respondents had tested for HIV previously, and the majority believed themselves to be either "definitely" or "probably" uninfected. There was evidence of continuing risk practices among some of those with known HIV infection.

Conclusions: This is the first estimate of actual and undiagnosed HIV infection among a community sample of gay men in New Zealand. While relatively low compared to other countries with mature epidemics, HIV prevalence was elevated in subgroups of MSM based on behaviour, and diagnosis rates varied by ethnicity. Prevention should focus on raising condom use and earlier diagnosis among those most at risk, and encouraging safe behaviour after diagnosis.
\end{abstract}

\section{Background}

Men who have sex with men (MSM) are the group most implicated in HIV transmission in New Zealand, accounting for over three-quarters of all locally acquired infections diagnosed between 1996 and 2008 [1]. Since 2000 , as in many developed countries, the annual number of new diagnoses in this group has increased [1]; in fact the largest number of MSM ever diagnosed in New

\footnotetext{
* Correspondence: peter.saxton@otago.ac.nz

'AIDS Epidemiology Group, Department of Preventive and Social Medicine, University of Otago Medical School, PO Box 913, Dunedin, New Zealand Full list of author information is available at the end of the article
}

Zealand was in 2010 [2]. Following the widespread introduction of effective antiretroviral treatment (ART) in 1996, life expectancy for people with HIV infection has improved $[3,4]$. Together with more new infections, reduced mortality has resulted in a doubling of the estimated number of MSM living with HIV in New Zealand between 1999 and 2009 [5]. This larger HIV prevalence pool would be expected to result in further increases in new infections unless there is a reduction in the annual transmission rate from infected MSM at a community level [3].

\section{Biomed Central}

(C) 2012 Saxton et al; BioMed Central Ltd. This is an Open Access article distributed under the terms of the Creative Commons Attribution License (http://creativecommons.org/licenses/by/2.0), which permits unrestricted use, distribution, and reproduction in any medium, provided the original work is properly cited. 
Early diagnosis can help reduce the infectivity of HIV positive individuals by motivating behaviour change and through timely antiretroviral treatment [6]. Those remaining undiagnosed, whether in the highly infectious early acute phase or prolonged latent phase of infection, play a disproportionate role in new HIV transmissions $[7,8]$. Mathematical modelling has suggested that in the Australian context, an estimated 9\% of HIV-infected MSM with undiagnosed HIV infection could account for approximately $31 \%$ of new annual transmissions [9].

While the prevalence of diagnosed HIV among MSM has been estimated from self report in behavioural surveillance in New Zealand [10] and routine surveillance of new diagnoses [5], the only previous studies that have measured the true prevalence of HIV in this group have been among attenders at sexual health clinics [11]. However, those findings may not be generaliseable to the broader population of MSM at risk of HIV.

The aim of this study is to provide for the first time measures of the prevalence of HIV infection and the proportion undiagnosed in a community sample of MSM in Auckland, New Zealand. We also believe this to be the first internationally to introduce oral fluid specimen collection to an existing programme of behavioural surveillance where most participants are recruited from a large community event, enabling comparable measures of HIV prevalence to be collected in the future.

\section{Methods}

Recruitment for the most recent Gay Auckland Periodic Sex Survey (GAPSS), a behavioural survey that has been undertaken every 2 years between 2002 and 2008 [10], took place in February 2011 at a gay community fair day in New Zealand's largest city Auckland, and over the subsequent week in the city's three gay bars and four sex-on-site venues. Participation criteria were having had sex with another man in the previous 5 years and being aged 16 or over. Sex was defined as "any physical contact you felt was sexual". At the gay community fair day, recruiters worked in teams from two large tents and approached men as they walked past. Recruiters invited men who agreed to self-complete the GAPSS questionnaire to also provide an oral fluid specimen using a specially designed collection device (OraSure Technologies, Inc., Bethlehem, PA, USA). All the main gay bars and sex-on-site venues in Auckland were approached and agreed to participate. In these venues, trained recruiters invited all men present to participate in the survey and to provide a specimen. Recruitment was scheduled over 3-4 h periods at different times of the week to generate a large and diverse sample. Both the questionnaire and oral fluid sample were anonymous, with the two linked by a non-identifying code.
An information sheet was provided to all participants that made it clear they would not receive their individual test result, and provided details of HIV testing services in Auckland. All participants were offered a lollipop as a token of appreciation. Prior to fieldwork we undertook consultation with a number of stakeholders including indigenous Maori. The study received ethics approval from the Northern $\times$ Regional Ethics Committee of the New Zealand Ministry of Health. Details of the recruitment phase are described in Additional file 1.

The questionnaire contained the core items used in previous GAPSS rounds covering sexual partnering, sexual behaviours and condom use, attitudes, knowledge, HIV testing and demographic variables [10]. Those who had ever been tested for HIV were asked the result of their most recent test; and all, including those who had never been tested, were asked what they believed their present HIV status to be. In 2011, new items were added on injecting drug use and ART use.

Oral fluid samples were tested by the National Reference Laboratory (NRL) in Melbourne, Australia using an in-house version of the bioMériuex Vironostika Oral Fluid GACELISA test kit that had been developed for a previous study [12]. Specimens were initially tested for total saliva immunoglobulin G (IgG). Samples with adequate IgG were screened for HIV antibodies with the GACELISA. Positive samples were rescreened and if repeatedly reactive underwent confirmatory Western blot testing. The sensitivity and specificity of this process is believed to be $100 \%$ (95\%CI: 95.0-100.0) and 100\% (95\%CI: 95.0-100.0) respectively [12].

The investigation of variations in HIV prevalence was informed by hypotheses that infection status would vary by patterns in exposure, by the underlying HIV prevalence among sexual contacts and by health seeking behaviours. Statistical analysis was performed using Stata version 11 [StataCorp, College Station, TX, USA). Pearson's chi-squared and Fisher's exact tests examined bivariate associations, and logistic regression was used to generate adjusted odds ratios (AORs) controlling for recruitment site, age and ethnicity.

\section{Results}

One thousand three hundred and eighteen questionnaires were returned from 3791 invitations to participate, an acceptance rate of $48.5 \%$ of men approached who were considered likely to be eligible. This was $49.7 \%$ at the fair day, $52.3 \%$ at the gay bars, and $40.7 \%$ at the sex-on-site venues. Fourteen questionnaires were subsequently excluded due to incomplete responses or ineligibility.

Recruiters obtained 1073 oral fluid specimens from survey respondents. Five either contained no oral fluid or had very low levels of IgG, resulting in 1068 testable 
samples. Of these, 67 were repeatedly reactive on GACELISA and had a positive Western blot, and four were repeatedly reactive on GACELISA but negative on Western blot, two of which were deemed positives as their laboratory optical density data were close to the cut-off and these men reported being HIV infected and on ART. One positive and two negative samples were unlabelled, and a further 14 negative samples either had no corresponding questionnaire or were from ineligible or discarded respondents. Hence there were 1049 linked samples and questionnaires, of which 68 were from men deemed infected with HIV.

\section{Specimen provision rate}

Collecting 1049 samples from 1304 eligible men resulted in an overall provision rate of oral fluid specimens of $80.4 \%$ enrolled in the survey. This varied by site, being highest at the fair day $(82.3 \%)$, and lower at the bars (71.5\%) and sex-on-site venues (76.5\%) (Table 1). While there was no difference in specimen provision by any of the demographic characteristics, it did differ by certain aspects of sexual behaviour in the previous 6 months. It was lower among men with only one partner (76.6\%), among those with more than 20 partners $(73.3 \%)$ and among those with no recent casual partner (76.4\%), and higher among those reporting unprotected anal intercourse with a casual partner (86.6\%) and among those who had had a sexually transmitted infection (STI) in the previous 12 months (88.6\%).

\section{HIV prevalence}

The overall prevalence of HIV infection was 6.5\% (95\% CI 5.1-8.1\%). This was lower in those aged $16-29$ years (3.3\%), compared to those aged 30-44 (7.5\%) and 45 years or older (8.9\%) (Table 2). After adjusting for ethnicity and site of enrolment, the OR for HIV infection was significantly higher for those aged $30-44$ years $(2.4,95 \%$ CI 1.2-4.9) and 45 years over (2.5, 95\% CI 1.2-4.9), compared to men under 30 . The prevalence was also higher among those resident overseas (13.7\%) than those living in Auckland (6.0\%) or elsewhere in New Zealand (4.8\%); the AOR for overseas residents was significantly raised (2.2, 95\% CI 1.1-4.8 adjusted for age, ethnicity and site of enrolment) compared to Auckland residents. There were no statistically significant differences by recruitment site, ethnicity, education or sexual identity.

There were a number of significant differences in HIV prevalence by recent risk behaviour after adjusting for recruitment site, age and ethnicity (Table 2). HIV prevalence rose with increasing numbers of sexual partners in the previous 6 months, being $3.5 \%$ and $3.6 \%$ respectively among those having none or one male sexual partner, 9.9\% among those with 6-20 male partners (AOR 2.6, 95\% CI 1.2-5.8 compared to one partner), and $12.9 \%$ among those with over 20 (AOR 3.5, 95\% CI 1.7-6.9 compared to one partner in that period). Prevalence was higher among those who had any unprotected anal sex with a casual male partner in the previous 6 months (11.9\%) (AOR 3.4, 95\% CI 1.7-6.9 compared to those who did not have casual sex) and among respondents who had had sex with a male partner they had met online in the previous six months (9.4\%) (AOR 2.3, 95\% CI 1.3-4.3 compared to those who had never met a partner in this way). HIV prevalence was also significantly higher among the small number of respondents who had injected drugs in the previous six months $(20.0 \%)$ (AOR 4.9, 95\% CI 1.3-18.9). Conversely, HIV prevalence was lower among respondents who had never tested for HIV in their lifetime (1.5\%) (AOR 0.24, 95\% CI 0.070.79 compared to those who had tested at least once).

\section{Undiagnosed HIV infection}

Of the 67 men with HIV infection who completed questions on past HIV testing and their most recent result, 53 (79.1\%) had been diagnosed with HIV. Hence 14 of the infected men $(20.9 \%, 95 \%$ CI $11.9-32.6)$ were unaware of their infection, a prevalence of unrecognised HIV of $1.3 \%(14 / 1046)$.

The prevalence of previously known and of unknown HIV infection, and the proportion diagnosed by demographic characteristics are described in Table 3 . The small number $(n=14)$ of respondents with undiagnosed HIV infection precludes formal comparison of the proportion diagnosed by all of these characteristics. However, significantly more of those of European ethnicity (90\%, 95\% CI 78-97) had been diagnosed compared to all those of any other ethnicity when combined (50\%, 95\% CI 25-75) (Fisher's exact $p=0.002$ ). This difference in diagnosis rates remained when limited to the $55 \mathrm{HIV}$ positive respondents normally resident in New Zealand (90\% among European versus 53\% among non-European, Fisher's exact $p=0.005$ ), a subset which is more relevant to informing HIV testing policy in this country.

\section{Characteristics of respondents without HIV and with known and unknown HIV infection}

Tables 4 and 5 provide a description of respondents without HIV and with known and unknown HIV infection. Formal statistical comparisons are only made between the former two groups in view of the small number of men with unknown infection $(n=14)$.

Table 4 shows the recent behaviour of the three respondent groups. Those with diagnosed HIV infection were significantly more likely than uninfected men to have had more than 20 partners in the past 6 months ( $17.3 \%$ versus $7.8 \%, p=0.03$ ), and to have had unprotected anal sex with casual partners $(42.3 \%$ versus $20.0 \%, p<0.001)$. Of the men with diagnosed HIV 
Table 1 Proportion of survey respondents who provided oral fluid specimens by recruitment site, demographic characteristics, sexual and other HIV risk and health seeking behaviour

\begin{tabular}{|c|c|c|c|c|}
\hline & \multirow{2}{*}{$\begin{array}{l}\text { Number } \\
\text { eligible }\end{array}$} & \multicolumn{2}{|c|}{ Specimen provided } & \multirow[t]{2}{*}{ p-value } \\
\hline & & $\mathrm{n}$ & $\%$ & \\
\hline Recruitment site & & & & 0.006 \\
\hline Fair day & 994 & 818 & 82.3 & \\
\hline Gay bars & 123 & 88 & 71.5 & \\
\hline Sex-on-site venues & 187 & 143 & 76.5 & \\
\hline \multicolumn{5}{|l|}{ Demographic characteristics } \\
\hline Age & & & & 0.52 \\
\hline $16-29$ & 454 & 367 & 80.8 & \\
\hline $30-44$ & 450 & 363 & 80.7 & \\
\hline $45+$ & 364 & 304 & 83.5 & \\
\hline Ethnicity & & & & 0.93 \\
\hline European & 922 & 750 & 81.3 & \\
\hline Maori & 136 & 111 & 81.6 & \\
\hline Pacific & 37 & 29 & 78.4 & \\
\hline Asian & 130 & 107 & 82.3 & \\
\hline Other & 48 & 37 & 77.1 & \\
\hline Highest education & & & & 0.23 \\
\hline Less than tertiary degree & 659 & 544 & 82.6 & \\
\hline Tertiary degree or higher & 602 & 481 & 79.9 & \\
\hline Sexual identity & & & & 0.61 \\
\hline Gay & 1122 & 908 & 80.9 & \\
\hline Bisexual & 135 & 105 & 77.8 & \\
\hline Other & 40 & 31 & 77.5 & \\
\hline Residence & & & & 0.43 \\
\hline Auckland & 1023 & 834 & 81.5 & \\
\hline Other New Zealand & 150 & 125 & 83.3 & \\
\hline Overseas & 95 & 73 & 76.8 & \\
\hline \multicolumn{5}{|l|}{ Sexual and HIV risk behaviour } \\
\hline Sex with women $<6$ months & & & & 0.16 \\
\hline No & 1196 & 969 & 81.0 & \\
\hline Yes & 101 & 76 & 75.3 & \\
\hline Number of male partners in previous 6 months & & & & 0.007 \\
\hline 0 & 68 & 57 & 83.8 & \\
\hline 1 & 401 & 307 & 76.6 & \\
\hline $2-5$ & 438 & 371 & 84.7 & \\
\hline $6-20$ & 232 & 192 & 82.8 & \\
\hline$>20$ & 116 & 85 & 73.3 & \\
\hline Sex with casual partners in previous 6 months & & & & 0.007 \\
\hline No casual partners & 436 & 333 & 76.4 & \\
\hline Casual partners - no anal sex & 189 & 158 & 83.6 & \\
\hline Casual partners - anal sex all protected & 367 & 292 & 79.6 & \\
\hline Casual partners - anal sex not all protected & 246 & 213 & 86.6 & \\
\hline Sex with current regular partner in previous 6 months & & & & 0.21 \\
\hline No current regular partner & 542 & 445 & 82.1 & \\
\hline Regular partner - no anal sex & 109 & 83 & 76.2 & \\
\hline
\end{tabular}


Table 1 Proportion of survey respondents who provided oral fluid specimens by recruitment site, demographic characteristics, sexual and other HIV risk and health seeking behaviour (Continued)

\begin{tabular}{|c|c|c|c|c|}
\hline Regular partner - anal sex all protected & 193 & 148 & 76.7 & \\
\hline Regular partner - anal sex not all protected & 391 & 325 & 83.1 & \\
\hline Injecting drug use & & & & 0.47 \\
\hline Never injected & 1167 & 950 & 81.4 & \\
\hline Injected at least once in lifetime & 90 & 76 & 84.4 & \\
\hline \multicolumn{5}{|l|}{ Health seeking behaviour } \\
\hline Sexual health check up in previous 12 months & & & & 0.86 \\
\hline No & 618 & 504 & 81.6 & \\
\hline Yes & 658 & 534 & 81.2 & \\
\hline STI in previous 12 months & & & 0.05 & \\
\hline No & 1150 & 930 & 80.9 & \\
\hline Yes & 105 & 93 & 88.6 & \\
\hline HIV testing history & & & 0.17 & \\
\hline Last tested HIV negative & 937 & 755 & 80.6 & \\
\hline Tested HIV positive & 74 & 55 & 74.3 & \\
\hline Never tested & 249 & 209 & 83.9 & \\
\hline Total & 1304 & 1049 & 80.4 & \\
\hline
\end{tabular}

Table omits data on respondents with missing information

infection who had engaged in unprotected anal intercourse with casual partners, a similar proportion were on ART $(64.8 \%)$ as those who did not have unprotected sex $(60.7 \%)(p=0.59)$. There were no differences in the proportion reporting a regular sexual partner nor the behaviour within this relationship between diagnosed positive and HIV negative respondents. However, men with known HIV infection who had any unprotected sex with a regular partner were much more likely to report that this partner was also HIV positive (68.4\% versus $2.3 \%, p<0.001)$. Men with diagnosed HIV were also more likely to have had sex with a man they had met on the Internet (44.2\% versus $28.9 \%, p=0.03)$, and to have injected drugs in the previous 6 months $(5.8 \%$ versus $1.3 \%, p=0.04)$. Similar proportions of men with known HIV infection (10.5\%) and uninfected men $(8.8 \%)$ had had an STI in the previous year.

Recent sexual behaviour of the men with undiagnosed HIV infection was not strikingly different from those with known infection (Table 4), but in view of the small number of the former statistical testing was not undertaken. Of these 14 respondents, 11 (78.6\%) had previously tested for HIV; three in the last 6 months, five between 6 and 11 months prior, and three more than a year prior. Most (12/14, 85.7\%) believed they were "definitely" or "probably" HIV negative at the time of survey, similar to the uninfected men (94.9\%). One believed he was "definitely positive" and one stated that he "didn't know".

\section{Attitudes}

Attitudes to HIV related issues are shown in Table 5 where again formal statistical comparisons are only made between the known infected and uninfected men. More of the former agreed that "HIV is less serious than it used to be" (52.8\% versus $30.5 \%, p=0.001)$. While the majority of men in both groups agreed that "condoms are OK as part of sex", this was reported by significantly more of the HIV negative men (96.7\% versus $90.6 \%, p=0.04)$. Uninfected men were more likely to agree that there was a "condom culture" among the men they have sex with $(75.7 \%$ versus $54.9 \%, p=0.001)$, and less likely to "sometimes feel under pressure to not use a condom" (28.8\% versus $45.3 \%, p=0.01)$. Furthermore, those with diagnosed HIV were less likely to agree that a man who knew he had HIV would disclose his HIV status before sex (18.9\% versus $38.4 \%, p=$ 0.006).

\section{Discussion and conclusions}

This is the first study to investigate HIV prevalence in a large, diverse community sample of gay men in New Zealand. Overall this was 6.5\%, and higher among older men and those living outside New Zealand. Prevalence was markedly elevated among those with more sexual partners in the previous 6 months, those who had had unprotected anal sex with a casual partner, who had met a sexual partner online or who had injected drugs in that period. About one fifth (20.9\%) of infected men 
Table 2 Prevalence of HIV infection and adjusted odds ratio by recruitment site, demographic characteristics, sexual and other HIV risk and health seeking behaviour

\begin{tabular}{|c|c|c|c|}
\hline & \multicolumn{2}{|c|}{ HIV prevalence } & \multirow[t]{2}{*}{ AOR $(95 \% \mathrm{Cl})$} \\
\hline & $\mathrm{n} / \mathrm{N}$ & $\%$ & \\
\hline \multicolumn{4}{|l|}{ Recruitment site } \\
\hline Fair day & $49 / 817$ & 6.0 & 1 \\
\hline Gay bars & $4 / 87$ & 4.6 & $0.69(0.24-2.0)$ \\
\hline Sex-on-site venues & $15 / 143$ & 10.5 & $1.6(0.85-3.2)$ \\
\hline \multicolumn{4}{|l|}{ Demographic characteristics } \\
\hline \multicolumn{4}{|l|}{ Age group } \\
\hline $16-29$ & $12 / 366$ & 3.3 & 1 \\
\hline $30-44$ & $27 / 362$ & 7.5 & $2.4(1.2-4.9)$ \\
\hline $45+$ & $27 / 304$ & 8.9 & $2.5(1.2-4.9)$ \\
\hline \multicolumn{4}{|l|}{ Ethnicity } \\
\hline European & $50 / 750$ & 6.7 & 1 \\
\hline Maori & $7 / 110$ & 6.4 & $1.1(0.47-2.5)$ \\
\hline Pacific & $3 / 29$ & 10.3 & $2.0(0.55-6.9)$ \\
\hline Asian & $3 / 107$ & 2.8 & $0.41(0.12-1.4)$ \\
\hline Other & $3 / 36$ & 8.3 & $1.4(0.41-4.8)$ \\
\hline \multicolumn{4}{|l|}{ Sexual identity } \\
\hline Gay & $64 / 906$ & 7.1 & 1 \\
\hline Bisexual or other & $4 / 136$ & 2.9 & $0.39(0.13-1.1)$ \\
\hline \multicolumn{4}{|l|}{ Highest education } \\
\hline Less than tertiary degree & $38 / 543$ & 7.0 & 1 \\
\hline Tertiary degree or higher & $26 / 480$ & 5.4 & $0.76(0.45-1.3)$ \\
\hline \multicolumn{4}{|l|}{ Residence } \\
\hline Auckland & $50 / 832$ & 6.0 & 1 \\
\hline Other New Zealand & $6 / 125$ & 4.8 & $0.78(0.32-1.9)$ \\
\hline Overseas & $10 / 73$ & 13.7 & $2.2(1.1-4.8)$ \\
\hline \multicolumn{4}{|l|}{ Sexual and HIV risk behaviour } \\
\hline \multicolumn{4}{|c|}{ Number of male partners in previous 6 months } \\
\hline 0 & $2 / 57$ & 3.5 & $0.54(0.07-4.3)$ \\
\hline 1 & $11 / 307$ & 3.6 & 1 \\
\hline $2-5$ & $22 / 369$ & 6.0 & $1.6(0.76-3.5)$ \\
\hline $6-20$ & $19 / 192$ & 9.9 & $2.7(1.2-5.8)$ \\
\hline$>20$ & $11 / 85$ & 12.9 & $3.5(1.4-8.7)$ \\
\hline \multicolumn{4}{|c|}{ Casual male partnerships in previous 6 months } \\
\hline No casual partners & $14 / 333$ & 4.2 & 1 \\
\hline Casual partners - no anal sex & $6 / 158$ & 3.8 & $0.88(0.32-2.4)$ \\
\hline Casual partners - anal sex all protected & 20/292 & 6.9 & $1.7(0.81-3.6)$ \\
\hline Casual partners - anal sex not all protected & $25 / 211$ & 11.9 & $3.4(1.7-6.9)$ \\
\hline \multicolumn{4}{|c|}{ Regular male partnerships in previous 6 months } \\
\hline No current regular partner & $26 / 445$ & 5.8 & 1 \\
\hline Regular partner - no anal sex & $3 / 83$ & 3.6 & $0.62(0.18-2.1)$ \\
\hline Regular partner - anal sex all protected & $12 / 148$ & 8.1 & $1.5(0.72-3.1)$ \\
\hline Regular partner - anal sex not all protected & $24 / 324$ & 7.4 & $1.4(0.75-2.5)$ \\
\hline \multicolumn{4}{|l|}{ Sex with a man met online } \\
\hline No & 20/385 & 5.2 & 1 \\
\hline Yes but not in previous 6 months & $18 / 339$ & 5.3 & $1.2(0.60-2.3)$ \\
\hline
\end{tabular}


Table 2 Prevalence of HIV infection and adjusted odds ratio by recruitment site, demographic characteristics, sexual and other HIV risk and health seeking behaviour (Continued)

\begin{tabular}{|c|c|c|c|}
\hline Yes in previous 6 months & $29 / 308$ & 9.4 & $2.3(1.2-4.3)$ \\
\hline \multicolumn{4}{|l|}{ Injecting drug use } \\
\hline No/not in previous 6 months & $63 / 1008$ & 6.3 & 1 \\
\hline Yes in previous 6 months & $3 / 15$ & 20.0 & $4.9(1.3-18.7)$ \\
\hline \multicolumn{4}{|l|}{ Health seeking behaviour } \\
\hline \multicolumn{4}{|c|}{ Sexually transmitted infection in the previous 12 months } \\
\hline$\overline{\text { No }}$ & $60 / 928$ & 6.5 & 1 \\
\hline Yes & $6 / 93$ & 6.5 & $0.93(0.38-2.2)$ \\
\hline \multicolumn{4}{|l|}{ HIV testing } \\
\hline Tested at least once in lifetime & $65 / 839$ & 7.8 & 1 \\
\hline Never tested & $3 / 202$ & 1.5 & $0.24(0.07-0.79)$ \\
\hline$\overline{T o t a l}$ & $68 / 1047$ & 6.5 & \\
\hline
\end{tabular}

$A O R$ adjusted odds ratio controlling for the effect of site, age and ethnicity $\mathrm{Cl}$ confidence interval

Table omits data on respondents with missing information

were unaware of their infection. A lower proportion of ethnically European men were undiagnosed compared to other respondents. The vast majority of undiagnosed men thought they were HIV negative. Diagnosed HIV positive men were more likely to report more than 20 sexual partners, unprotected anal sex with casual partners, sex with a man they met online, and injecting drugs in the previous 6 months compared to HIV negative respondents. Uninfected men were more likely to exhibit attitudes conducive to HIV control.

We have demonstrated the acceptability of adding an anonymous oral fluid collection component into HIV behavioural surveillance undertaken at a large gay community event as well as gay bars and sex-on-site venues. The overall high specimen provision rate $(80.4 \%)$ with little variation across enrolment sites, age and ethnicity suggest that the HIV prevalence estimates will be representative of those men taking part in the behavioural survey. As the survey is repeated in a consistent manner over time this will enable a comparable measure of HIV prevalence to be obtained in the future.

There are several limitations to the study. It is not possible to be certain of the actual response rate to the questionnaire as those who initially refused might have completed it subsequently, but it is probably in the order of half invited. The recruitment occurred in a number of community settings in Auckland so the findings may not be generalised to all gay men in New Zealand, to MSM in Auckland who do not attend these settings, or who only seek sexual partners through the Internet. Specimen provision was higher among men who had recently engaged in unprotected anal intercourse which may have resulted in slightly overestimating HIV prevalence among all study participants. While behavioural data rely on self-report that cannot be verified, the anonymity of the questionnaire and study protocols present little incentive to misreport. A small number of responses and biological data were inconsistent, with four respondents whose specimens were HIV negative on Western blot indicating they had tested HIV positive in their questionnaire. Two of the latter were deemed positive on the basis of them having relatively high optical densities on the original GACELISA test and reporting being on ART.

A prevalence of $6.5 \%$ in this Auckland study of MSM is consistent with past New Zealand clinic studies. In the most recent 2005/6 unlinked anonymous study of HIV among sexual health clinic attenders, the prevalence among MSM in Auckland was 6.1\% [11]. Those who attend sexual health clinics would be expected to have a higher prevalence than those in a community sample as they have self identified as having STI risk. However, HIV prevalence in the latter is likely to have increased in recent years, with more infections having occurred coupled with prolonged survival.

Table 6 summarises the results from a number of prevalence studies among MSM in community settings internationally, with a wide range of findings. When placed alongside these, our data on HIV prevalence show that HIV remains relatively well controlled among Auckland MSM, despite having a mature epidemic that has existed since the early 1980s. This is consistent with the rate of new HIV diagnoses among MSM being lower in New Zealand than that in Australia, the United Kingdom and the USA [1]. As the rate of HIV diagnosis is higher in Auckland than elsewhere in New Zealand this conclusion will be valid for the whole country. 
Table 3 Prevalence of diagnosed and undiagnosed HIV infection, and proportion (95\% confidence interval) of HIV infections diagnosed, by recruitment site and demographic characteristics

\begin{tabular}{|c|c|c|c|c|}
\hline & Total & $\begin{array}{c}\text { Diagnosed HIV } \\
\text { n (\%) }\end{array}$ & $\begin{array}{c}\text { Undiagnosed } \\
\text { HIV n (\%) }\end{array}$ & $\begin{array}{c}\text { Proportion } \\
\text { diagnosed } \%(95 \% \mathrm{Cl})\end{array}$ \\
\hline \multicolumn{5}{|l|}{ Recruitment site } \\
\hline Fair day & 816 & $39(4.8)$ & $9(1.1)$ & $81(67-91)$ \\
\hline Gay bar & 87 & $4(4.6)$ & $0(0.0)$ & $100(40-100)$ \\
\hline Sex-on-site venue & 143 & $10(7.0)$ & $5(3.5)$ & $67(38-88)$ \\
\hline \multicolumn{5}{|l|}{ Age group } \\
\hline $16-29$ & 366 & $7(1.9)$ & $5(1.4)$ & $58(28-85)$ \\
\hline $30-44$ & 361 & $22(6.1)$ & $4(1.1)$ & $85(65-96)$ \\
\hline $45+$ & 304 & $23(7.6)$ & $4(1.3)$ & $85(66-96)$ \\
\hline \multicolumn{5}{|l|}{ Ethnicity } \\
\hline European & 749 & $44(5.9)$ & $5(0.7)$ & $90(78-97)$ \\
\hline Maori & 110 & $4(3.6)$ & $3(2.7)$ & $57(18-90)$ \\
\hline Pacific Island & 29 & $1(3.5)$ & $2(6.9)$ & $33(1-91)$ \\
\hline$\overline{A s i a n}$ & 107 & $0(0.0)$ & $3(2.8)$ & $0(0-71)$ \\
\hline Other & 36 & $3(8.3)$ & $0(0.0)$ & $100(29-100)$ \\
\hline \multicolumn{5}{|l|}{ Sexual identity } \\
\hline Gay & 905 & $51(5.6)$ & $12(1.3)$ & $81(69-90)$ \\
\hline Bisexual or other & 136 & $2(1.9)$ & $2(1.9)$ & $50(7-93)$ \\
\hline \multicolumn{5}{|l|}{ Highest education } \\
\hline Less than tertiary degree & 542 & $34(6.3)$ & $3(0.6)$ & $92(78-98)$ \\
\hline Tertiary degree or higher & 480 & $17(3.5)$ & $9(1.9)$ & $65(44-83)$ \\
\hline \multicolumn{5}{|l|}{ Residence } \\
\hline Auckland & 831 & $38(4.6)$ & $11(1.3)$ & $78(63-88)$ \\
\hline Other New Zealand & 125 & $6(4.8)$ & $0(0.0)$ & $100(54-100)$ \\
\hline Overseas & 73 & $8(11.0)$ & $2(2.7)$ & $0(44-97)$ \\
\hline Total & 1046 & $53(5.1)$ & $14(1.3)$ & 79.1 \\
\hline
\end{tabular}

Cl confidence interval

Table omits data on respondents with missing information

The proportion of HIV infected MSM who were undiagnosed (20.9\%) in Auckland is considerably lower than found previously in Melbourne [12], five UK cities [13-15] and 21 US cities [17] (Table 6). This is somewhat surprising as behavioural surveillance shows that HIV testing rates among gay men in Auckland [10] are not higher than in those cities $[14,17,20]$. This may indicate that testing in Auckland is targeting MSM at highest risk, which since 2006 has included rapid HIV testing services [21], or the incidence is lower in Auckland.

Unlike the experience in other countries [22-24], we found no statistically significant differences in overall HIV prevalence by ethnicity in our sample. In particular, HIV prevalence was the same in European (6.7\%) and in Māori (6.4\%) MSM. This is consistent with previously reported epidemiological data suggesting no overrepresentation of HIV in this group, but it is different to the experience of indigenous individuals within MSM communities elsewhere [25]. We did on the other hand find that HIV positive MSM of non-European ethnicity were less likely to have had been diagnosed. This finding is consistent with a recent analysis that non-European MSM are more likely to present late in the course of infection in New Zealand [26] and warrants further investigation.

In spite of this favourable position internationally, the number of new diagnoses among MSM in New Zealand continues at a higher level than in the late 1990s [1]. Findings from this study add to our understanding of HIV transmission and acquisition risks, and have implications for ongoing prevention. We believe four issues should be highlighted.

First, we have shown high prevalence of HIV among MSM who had recently engaged in certain behaviours, including having over 20 recent sexual partners, having unprotected anal intercourse with casual partners, meeting sexual partners online, and injecting drugs. Men 
Table 4 Behavioural characteristics of respondents without HIV infection, with known and with unknown HIV infection

\begin{tabular}{|c|c|c|c|}
\hline & \multirow{2}{*}{$\begin{array}{c}\text { Uninfected } \\
\text { n (\%) }\end{array}$} & \multicolumn{2}{|c|}{ HIV infected } \\
\hline & & $\begin{array}{c}\text { Known } \\
\text { n (\%) }\end{array}$ & $\begin{array}{c}\text { Unknown } \\
\text { n (\%) }\end{array}$ \\
\hline \multicolumn{4}{|c|}{ Number of male partners in previous 6 months } \\
\hline Up to 20 & $871(92.2)$ & $43(82.7)^{*}$ & $10(83.3)$ \\
\hline$>20$ & $74(7.8)$ & $9(17.3)$ & $2(16.7)$ \\
\hline \multicolumn{4}{|c|}{ Casual male partnerships in previous 6 months } \\
\hline No casual partners & $319(34.3)$ & $11(21.2) \dagger$ & $3(25.0)$ \\
\hline Casual partners - no anal sex & $152(16.4)$ & $6(11.5)$ & $0(0)$ \\
\hline Casual partners - anal sex all protected & $272(29.3)$ & $13(25.0)$ & $6(50.0)$ \\
\hline Casual partners - anal sex not all protected & $186(20.0)$ & $22(42.3)$ & $3(25.0)$ \\
\hline \multicolumn{4}{|c|}{ Regular male partnerships in previous 6 months } \\
\hline No regular partner & $419(44.8)$ & $22(42.3)$ & $4(33.3)$ \\
\hline Regular partner - no anal sex & $80(8.6)$ & $2(3.9)$ & $1(8.3)$ \\
\hline Regular partner - anal sex all protected & $136(14.6)$ & $9(17.3)$ & $2(16.7)$ \\
\hline Regular partner - anal sex not all protected & $300(32.1)$ & $19(36.5)$ & $5(41.7)$ \\
\hline \multicolumn{4}{|l|}{ Sex with a man met online } \\
\hline No & $365(37.8)$ & $16(30.8) \ddagger$ & $4(28.6)$ \\
\hline Yes but not $<6$ months & $321(33.3)$ & $13(25.0)$ & $4(28.6)$ \\
\hline Yes $<6$ months & $279(28.9)$ & $23(44.2)$ & $6(42.9)$ \\
\hline \multicolumn{4}{|l|}{ Injecting drug use } \\
\hline No & $897(93.7)$ & $38(73.1) \neq$ & $12(85.7)$ \\
\hline Yes but not $<6$ months & $48(5.0)$ & $11(21.2)$ & $2(14.3)$ \\
\hline Yes $<6$ months & $12(1.3)$ & $3(5.8)$ & $0(0)$ \\
\hline \multicolumn{4}{|c|}{ Sexually transmitted infection in previous 12 months } \\
\hline No & $847(91.2)$ & $44(89.8)$ & $13(92.9)$ \\
\hline Yes & $82(8.8)$ & $5(10.2)$ & $1(7.1)$ \\
\hline Total & $979(100.0)$ & $53(100.0)$ & $14(100.0)$ \\
\hline
\end{tabular}

* $p<0.05$ for comparison between uninfected and known infected

$\dagger p<0.001$ for comparison between uninfected and known infected on dichotomised variable (last category versus rest)

$\neq p<0.05$ for comparison between uninfected and known infected on dichotomised variable (first category versus rest)

Table omits data on respondents with missing information

with these behaviours can act as ongoing reservoirs of infection especially if condoms are not used for anal sex, creating clusters of transmission if sexual contact is assortative (sexual mixing occurs with similar men), or dispersing HIV through sexual networks if contact patterns are dissortative. As most individuals will have imperfect information about the past behaviour of a prospective sexual partner, choosing to have unprotected anal intercourse may entail more risk than anticipated if this decision is based on an assumption that HIV prevalence is relatively low among all MSM.

Secondly, the majority of the men with undiagnosed HIV infection believed themselves to be HIV negative. Many had had a quite recent HIV test, and may consequently have held strong but incorrect convictions about their absence of HIV infection. These findings challenge safe sex strategies based on disclosure of HIV status alone. This is emphasised by our result that most of the diagnosed positive men would not expect a man who knew he had HIV to disclose this before sex; possibly a reflection of their own experience. Conversely the vast majority of all participants reported that condoms were "OK as part of sex", although the proportion disagreeing with this was higher (9.4\%) among the known positive respondents. As condoms are a verifiable intervention by all participants during sex, they should continue to be strongly promoted.

Thirdly, the proportion undiagnosed varied among those with HIV. This was higher among men of nonEuropean ethnicity, and also - although chance could not be excluded as the explanation for this finding among those aged under 30 years. The reason for this needs to be explored further. Promotion of HIV testing should continue, and the responsiveness of testing 
Table 5 Responses to questions on attitudes to HIV-related issues of respondents without HIV infection, with known and with unknown HIV infection

\begin{tabular}{|c|c|c|c|}
\hline & \multirow{2}{*}{$\begin{array}{c}\text { Uninfected } \\
\text { n (\%) }\end{array}$} & \multicolumn{2}{|c|}{ HIV infected } \\
\hline & & $\begin{array}{c}\text { Known } \\
\text { n (\%) }\end{array}$ & $\begin{array}{c}\text { Unknown } \\
\text { n (\%) }\end{array}$ \\
\hline \multicolumn{4}{|c|}{ Condoms are OK as part of sex } \\
\hline Agree & $935(96.7)$ & $48(90.6)^{*}$ & $12(100.0)$ \\
\hline Disagree & $32(3.3)$ & $5(9.4)$ & $0(0)$ \\
\hline \multicolumn{4}{|c|}{ HIV/AIDS is a less serious threat than it used to be because of new treatments } \\
\hline Agree & $296(30.5)$ & $28(52.8) \dagger$ & $3(23.1)$ \\
\hline Disagree & $675(69.5)$ & $25(47.2)$ & $10(76.9)$ \\
\hline \multicolumn{4}{|c|}{ I would sometimes rather risk HIV transmission than use a condom during anal sex } \\
\hline Agree & $135(13.9)$ & $7(13.2)$ & $0(0)$ \\
\hline Disagree & $838(86.1)$ & $46(86.8)$ & $12(100.0)$ \\
\hline \multicolumn{4}{|c|}{ I don't like wearing condoms because they reduce sensitivity } \\
\hline Agree & $378(39.1)$ & $28(52.8)$ & $1(8.3)$ \\
\hline Disagree & $588(60.9)$ & $25(47.2)$ & $11(91.7)$ \\
\hline \multicolumn{4}{|c|}{ The sex I have is always as safe as I want it to be } \\
\hline Agree & $886(91.3)$ & $46(86.8)$ & $10(83.3)$ \\
\hline Disagree & $84(8.7)$ & $7(13.2)$ & $2(16.7)$ \\
\hline \multicolumn{4}{|c|}{ Sometimes I feel under pressure to not use a condom } \\
\hline Agree & $279(28.8)$ & $24(45.3) \neq$ & $3(25.0)$ \\
\hline Disagree & $690(71.2)$ & $29(54.7)$ & $9(75.0)$ \\
\hline \multicolumn{4}{|c|}{ I'd like to be better informed about HIV transmission risk } \\
\hline Agree & $734(75.8)$ & $35(67.3)$ & $10(76.9)$ \\
\hline Disagree & $234(24.2)$ & $17(32.7)$ & $3(23.1)$ \\
\hline \multicolumn{4}{|c|}{ I would never be willing to use condoms for anal sex } \\
\hline Agree & $94(9.7)$ & $7(13.2)$ & $0(0)$ \\
\hline Disagree & $875(90.3)$ & $46(86.8)$ & $12(100.0)$ \\
\hline \multicolumn{4}{|c|}{ There is a "condom culture" among the men I have sex with } \\
\hline Agree & $708(75.7)$ & $27(54.9) \dagger$ & $7(58.3)$ \\
\hline Disagree & $227(24.3)$ & $24(47.1)$ & $5(41.7)$ \\
\hline \multicolumn{4}{|c|}{ A man who knows he has HIV would tell me he was positive before we had sex } \\
\hline Agree & $371(38.4)$ & $10(18.9) \neq$ & $5(41.7)$ \\
\hline Disagree & 595 (61.6) & $43(81.1)$ & $7(58.3)$ \\
\hline Total & $979(100.0)$ & $53(100.0)$ & $14(100.0)$ \\
\hline
\end{tabular}

Agree = "strongly agree" or "agree" with statement, Disagree = "strongly disagree" or "disagree" with statement

* $p<0.05$ for comparison between uninfected and known infected

$\dagger p=0.001$ for comparison between uninfected and known infected

$\neq p<0.01$ for comparison between uninfected and known infected

Table omits data on respondents with missing information

services to diverse groups of MSM encouraged. All MSM with HIV infection should be able to access treatment as early as appropriate, which will not only be to their own benefit but also potentially reduce ongoing spread. More HIV testing, in terms of both coverage and frequency, should not however be considered the panacea for HIV control. The benefits of earlier diagnosis on ongoing transmission can only be expected if behaviour changes, and/or treatment that reduces infectivity is begun. HIV is particularly infectious in the weeks after infection, and modelling has shown that individuals in this primary stage of infection transmit disproportionately to their number in the community, but even very frequent routine testing would pick up few individuals at this stage. A negative HIV test should be seen as an opportunity to discuss HIV risk-taking, and how this could be reduced. To diagnose HIV infection after a number of negative 
Table 6 HIV antibody prevalence and undiagnosed infection in community samples of MSM in selected countries

\begin{tabular}{|c|c|c|c|c|c|c|}
\hline Location & Year conducted & Recruitment sites & Sample size* & HIV prevalence (\%) & Proportion undiagnosed (\%) & Ref \\
\hline \multirow[t]{3}{*}{ England (3 cities) } & $2003-4$ & $\mathrm{~GB}, \mathrm{GS}$ & 348 & 8.6\% Manchester & 36.7\% Manchester & {$[13,14]$} \\
\hline & & & 1436 & 12.3\% London & 44.1\% London & \\
\hline & & & 373 & 13.7\% Brighton & 33.3\% Brighton & \\
\hline \multirow[t]{2}{*}{ Scotland (2 cities) } & 2005 & GB, GS & 749 & 3.6\% Glasgow & $48.1 \%$ Glasgow & {$[14,15]$} \\
\hline & & & 601 & 5.5\% Edinburgh & 36.4\% Edinburgh & \\
\hline Southern Africa (3 countries) & 2008 & SB & 537 & $17.4 \%$ & $76.3 \%$ & {$[16]$} \\
\hline \multirow[t]{3}{*}{ United States (21 cities) } & 2008 & $\mathrm{~GB}, \mathrm{SO}$ & 8153 & $19.2 \%$ overall & $43.5 \%$ overall & {$[17]$} \\
\hline & & & & 6.4\% Atlanta (low) & 15.4\% Seattle (low) & \\
\hline & & & & 37.8\% Baltimore (high) & 73.4\% Baltimore (high) & \\
\hline \multirow[t]{2}{*}{ Australia (2 cities) } & 2008 & GB, GS & 465 & 8.8\% Brisbane & 19.5\% Brisbane & {$[18]$} \\
\hline & & & 639 & 9.5\% Melbourne & 31.1\% Melbourne & {$[12]$} \\
\hline France (Paris) & 2009 & $\mathrm{~GB}, \mathrm{GS}$ & 886 & $17.7 \%$ & $20 \%$ & \\
\hline New Zealand (Auckland) & 2011 & $\begin{array}{l}\mathrm{GB}, \mathrm{GS}, \\
\mathrm{CE}\end{array}$ & 1049 & $6.5 \%$ & $20.9 \%$ & [19] \\
\hline
\end{tabular}

*Number providing viable oral fluid sample

GB gay bars/club, GS gay saunas/sex-on-site venues, $S B$ snowball sampling, SO social organisations, CE large community event

tests should be seen as a failure to make full use of these opportunities.

Fourthly, recent behaviour that increases the probability of HIV transmission was more common among MSM with diagnosed HIV than among respondents who were uninfected. Risk of transmission exists, especially if individuals are not on ART $[6,8]$, as was the case with $35.4 \%$ of diagnosed men in this study, or if HIV positive men have another STI [27], as was reported by $10 \%$ of our respondents in the previous year. We do not know if the behaviour of these individuals has changed since their HIV diagnosis, as would be suggested from previous overseas studies $[28,29]$, but it does raise concern about ongoing spread by some diagnosed men in New Zealand. As ART was not reported universally among those with known HIV it is unlikely these men had all engaged in unprotected anal intercourse believing themselves to be uninfectious. Further research is needed to better understand these experiences. Overall, these findings suggest that the risk of onward transmission of HIV should continue to be emphasised by those providing care to MSM with HIV, and by their support communities.

\section{Additional material}

Additional file 1: Methods and fieldwork. PDF file (text, table and figures), describes the planning, protocols and recruitment phase of the study.

\section{Acknowledgements}

The authors would like to thank the men who participated in the study and the venue operators for allowing us access. This research was funded by a
Health Research Council of New Zealand grant \#10-418. The AIDS Epidemiology Group at the University of Otago Medical School and the Research Unit at the New Zealand AIDS Foundation are funded by the Ministry of Health. PS conducted the research during tenure of a Health Sciences Career Development Award of the University of Otago. We also thank NRL in Melbourne, Australia for conducting the specimen testing.

\section{Author details}

${ }^{1}$ AIDS Epidemiology Group, Department of Preventive and Social Medicine University of Otago Medical School, PO Box 913, Dunedin, New Zealand. ${ }^{2}$ Research Unit, New Zealand AIDS Foundation, PO Box 6663, Wellesley Street, Auckland, New Zealand.

\section{Authors' contributions}

PS co-conceived the study, took overall lead on the project, analysed the GAPSS behavioural data and wrote the first draft. ND co-conceived the study and provided guidance on study design and interpretation. RG coordinated the oral fluid specimen collection. AH participated in study design and data collection. JR coordinated the GAPSS behavioural data collection. All authors read and approved the final manuscript.

\section{Competing interests}

The authors declare that they have no competing interests.

Received: 6 November 2011 Accepted: 1 February 2012

Published: 1 February 2012

\section{References}

1. Saxton PJ, Dickson NP, McAllister SM, Sharples K, Hughes AJ: Increase in HIV diagnoses among men who have sex with men in New Zealand from a stable low period. Sex Health 2011, 8:311-318.

2. AIDS Epidemiology Group: AIDS - New Zealand. Dunedin: University of Otago; 2011, 67.

3. May M, Sterne JA, Sabin C, Costagliola D, Justice AC, Thiebaut R, Gill J, Phillips A, Reiss P, Hogg R, et al: Prognosis of HIV-1-infected patients up to 5 years after initiation of HAART: collaborative analysis of prospective studies. AIDS 2007, 21:1185-1197.

4. HIV-CAUSAL Collaboration: The effect of combined antiretroviral therapy on the overall mortality of HIV-infected individuals. AIDS 2010, 24:123-137.

5. Saxton PJW, Dickson NP, McAllister SM, Hughes AJ, Sharples K: HIV prevalence among men who have sex with men in New Zealand 19852009: 25 years of public health monitoring. Int J STD AIDS, DOI: 10.1258/ ijsa.2011.011213. 
6. Cohen MS, Chen YQ, McCauley M, Gamble T, Hosseinipour MC, Kumarasamy N, Hakim JG, Kumwenda J, Grinsztejn B, Pilotto JH, et al: Prevention of HIV-1 infection with early antiretroviral therapy. N Engl J Med 2011, 365:493-505.

7. Miller WC, Rosenberg NE, Rutstein SE, Powers KA: Role of acute and early HIV infection in the sexual transmission of HIV. Curr Opin HIV AIDS 2010, 5:277-282.

8. Baggaley RF, White RG, Boily M-C: HIV transmission risk through anal intercourse: systematic review, meta-analysis and implications for HIV prevention. Int J Epidemiol 2010, 39:1048-1063.

9. Wilson DP, Hoare A, Regan DG, Law MG: Importance of promoting HIV testing for preventing secondary transmissions: modelling the Australian HIV epidemic among men who have sex with men. Sex Health 2009, 6:19-33.

10. Saxton P, Dickson N, Hughes A: GAPSS 2008: Findings from the Gay Auckland Periodic. Sex Survey Auckland: New Zealand AIDS Foundation; 2010.

11. McAllister SM, Dickson NP, Sharples K, Reid MR, Morgan JM, MacDonald EJ, Coughlan E, Johnston TM, Tanner NA, Paul C: Unlinked anonymous HIV prevalence among New Zealand sexual health clinic attenders: 20052006. Int J STD AIDS 2008, 19:752-757.

12. Pedrana AE, Hellard ME, Wilson K, Guy R, Stoove M: High rates of undiagnosed HIV infections in a community sample of gay men in Melbourne, Australia. J Acquir Immune Defic Syndr 2012, 59:94-99.

13. Dodds JP, Johnson AM, Parry JV, Mercey DE: A tale of three cities: persisting high HIV prevalence, risk behaviour and undiagnosed infection in community samples of men who have sex with men. Sex Transm Infect 2007, 83:392-396.

14. Williamson LM, Dodds JP, Mercey DE, Hart GJ, Johnson AM: Sexual risk behaviour and knowledge of HIV status among community samples of gay men in the UK. AIDS 2008, 22:1063-1070.

15. Williamson LM, Hart GJ: HIV prevalence and undiagnosed infection among a community sample of gay men in Scotland. J Acquir Immune Defic Syndr 2007, 45:224-230.

16. Baral S, Trapence G, Motimedi F, Umar E, lipinge S, Dausab F, Beyrer C: HIV prevalence, risks for HIV infection, and human rights among men who have sex with men (MSM) in Malawi, Namibia, and Botswana. PLoS One 2009, 4:e4997.

17. CDC: Prevalence and awareness of HIV infection among men who have sex with men - 21 cities, United States, 2008. MMWR 2010, 59:1201-1207.

18. Birrell F, Staunton S, Debattista J, Roudenko N, Rutkin W, Davis C: Pilot of non-invasive (oral fluid) testing for HIV within a community setting. Sex Health 2010, 7:11-16.

19. Barin F, Velter A, Boutssou A, Guinard J, Plantier JC, Vu SL, Pillonel J, Alexandre A, Semaille C: High prevalence of HIV-1 infection and diversity of circulating strains among men who have sex with men recruited in commercial gay venues in Paris (France): PREVAGAY 2009 Survey., 18th Conference on Retroviruses and Opportunistic Infections (CROI) 2011, Boston. Abstract\#:W-139.

20. Prestage G, Jin F, Zablotska IB, Imrie J, Grulich AE, Pitts M: Trends in HIV testing among homosexual and bisexual men in eastern Australian states. Sex Health 2008, 5:119-123.

21. Smythe E: Evaluation of 3 Month Auckland Based Rapid Test Pilot. Auckland: New Zealand AIDS Foundation; 2007.

22. Millet GA, Peterson JL, Wolitski RJ, Stall R: Greater risk for HIV infection of black men who have sex with men: a critical literature review. Am J Public Health 2006, 96:1007-1019.

23. Dougan S, Elford J, Rice B, Brown AE, Sinka K, Evans BG, Gill ON, Fenton KA Epidemiology of HIV among black and minority ethnic men who have sex with men in England and Wales. Sex Transm Infect 2005, 81:345-350.

24. Hickson F, Reid D, Weatherburn P, Stephens M, Nutland W, Boakye P: HIV, sexual risk, and ethnicity among men in England who have sex with men. Sex Transm Infect 2004, 80:443-450.

25. Shea B, Aspin C, Ward J, Archibold C, Dickson N, McDonald A, Penehira M, Halverson J, Masching R, McAllister S, Smith L, Kaldor J, Andersson N: HIV diagnoses in indigenous peoples: comparison of Australia, Canada and New Zealand. Int Health 2011, 3:193-198.

26. Dickson NP, McAllister SM, Sharples K, Paul C: Late presentation of HIV infection among adults in New Zealand - 2005-2010. HIV Medicine, Epub ahead of print 3 Nov 2011
27. Galvin SR, Cohen MS: The role of sexually transmitted diseases in HIV transmission. Nat Rev Microbiol 2004, 2:33-42.

28. Marks G, Crepaz N, Senterfitt JW, Janssen RS: Meta-analysis of high-risk sexual behavior in persons aware and unaware they are infected with HIV in the United States: implications for HIV prevention programs. J Acquir Immune Defic Syndr 2005, 39:446-453

29. Fox J, White PJ, Macdonald N, Weber J, McClure M, Fidler S, Ward H: Reductions in HIV transmission risk behaviour following diagnosis of primary HIV infection: a cohort of high-risk men who have sex with men. HIV Med 2009, 10:432-438.

Pre-publication history

The pre-publication history for this paper can be accessed here: http://www.biomedcentral.com/1471-2458/12/92/prepub

doi:10.1186/1471-2458-12-92

Cite this article as: Saxton et al:: Actual and undiagnosed HIV

prevalence in a community sample of men who have sex with men in Auckland, New Zealand. BMC Public Health 2012 12:92.

\section{Submit your next manuscript to BioMed Central and take full advantage of:}

- Convenient online submission

- Thorough peer review

- No space constraints or color figure charges

- Immediate publication on acceptance

- Inclusion in PubMed, CAS, Scopus and Google Scholar

- Research which is freely available for redistribution 